Purdue University

Purdue e-Pubs

CTRC Research Publications

Cooling Technologies Research Center

2015

\title{
Spurious Current Suppression in VOF-CSF Simulation of Slug Flow through Small Channels
}

Z.Pan

Purdue University

J.A. Weibel

Purdue University, jaweibel@purdue.edu

S V. Garimella

Purdue University, sureshg@purdue.edu

Follow this and additional works at: http://docs.lib.purdue.edu/coolingpubs

Pan, Z.; Weibel, J. A.; and Garimella, S V., "Spurious Current Suppression in VOF-CSF Simulation of Slug Flow through Small Channels" (2015). CTRC Research Publications. Paper 243.

http://dx.doi.org/10.1080/10407782.2014.916109

This document has been made available through Purdue e-Pubs, a service of the Purdue University Libraries. Please contact epubs@purdue.edu for additional information. 


\section{Spurious Current Suppression in VOF-CSF Simulation of Slug Flow through Small Channels ${ }^{1}$}

Zhenhai Pan, Justin A. Weibel, and Suresh V. Garimella ${ }^{2}$

School of Mechanical Engineering and Birck Nanotechnology Center

Purdue University, 585 Purdue Mall, West Lafayette, IN 47907 USA

${ }^{1}$ Submitted for possible publication in Numerical Heat Transfer A, December 2013

${ }^{2}$ Corresponding author: Tel. 1765494 5621; sureshg@purdue.edu 


\section{Running Title: Spurious Current Suppression in VOF-CSF}

A numerical treatment is proposed to minimize the creation of unphysical, spurious currents in modeling liquid-gas slug flow using the volume of fluid-continuum surface force (VOF-CSF) method. An elongated gas slug drawn into a small circular channel initially filled with liquid is considered. To suppress spurious currents formed by numerical errors in calculation of the surface tension force at small Capillary numbers $(C a<0.01)$, an artificial relative reference frame is specified with motion in a direction opposite that of the flow. An increase in the local relative velocity magnitude near the interface is demonstrated to be the key mechanism for spurious current suppression. A comparison of simulations performed with and without this treatment shows that spurious currents are eliminated at $C a=0.0029$; liquid film thickness, gas slug velocity, and liquid-phase circulation near the leading slug interface are preserved and the computed values agree with the literature. This demonstrates that the proposed moving reference frame method does not influence the computed physical phenomena of interest while suppressing unphysical spurious velocities.

Keywords: spurious current, volume of fluid, continuum surface force, slug flow, liquid film, microchannel 


\section{Nomenclature}

$\begin{array}{llll}C & \text { volume fraction } & \text { Greek } & \\ C a & \text { Capillary number, } C a=\mu U / \sigma & \delta & \text { film thickness }(\mathrm{m}) \\ C o & \text { Courant number, } C o=U \Delta t / \Delta x & \delta_{s} & \text { delta function } \\ D & \text { channel diameter }(\mathrm{m}) & \mu & \text { dynamic viscosity }\left(\mathrm{N} \mathrm{s} / \mathrm{m}^{2}\right) \\ F & \text { volumetric body force }\left(\mathrm{N} / \mathrm{m}^{3}\right) & \kappa & \text { interface curvature }\left(\mathrm{m}^{-1}\right) \\ L & \text { channel length }(\mathrm{m}) & \rho & \text { density }\left(\mathrm{kg} / \mathrm{m}^{3}\right) \\ \vec{n} & \text { unit normal vector } & \sigma & \text { surface tension }(\mathrm{N} / \mathrm{m}) \\ P & \text { pressure }\left(\mathrm{N} / \mathrm{m}^{2}\right) & \omega & \text { nondimensional vorticity } \\ R e & \text { Reynolds number, } R e=\rho U D / \mu & \text { Subscript } & \\ T & \text { time }(\mathrm{s}) & 0 & \text { static reference } \\ U & \text { velocity }(\mathrm{m} / \mathrm{s}) & S & \text { gas slug } \\ \vec{U} & \text { velocity vector }(\mathrm{m} / \mathrm{s}) & l & \text { liquid } \\ \Delta t & \text { time step }(\mathrm{s}) & r & \text { relative } \\ \Delta x & \text { mesh size }(\mathrm{m}) & r e f & \text { reference frame } \\ & & s & \text { surface tension }\end{array}$

\section{Introduction}

Liquid-gas slug flow in small capillary tubes and microchannels is important in heat transfer applications [1-4], micro-reaction systems [5,6], and microfluidic control [7,8]. Bretherton [9] pioneered theoretical analysis of single bubble motion in a rigid round channel under a lubrication assumption that at low Capillary numbers, viscous forces only affect the static interface profile near the wall. The dimensionless liquid film thickness around the bubble was found to be a function of only the Capillary number $\left(\delta / R=1.34 \mathrm{Ca}^{2 / 3}\right.$, validated experimentally for $10^{-4}<\mathrm{Ca}<10^{-2}$ ). Aussillous and Quéré [10] subsequently proposed a correlation with an extended range by fitting experimental data of their own and from Taylor [11]. Their correlation agreed with the experiments even at very high $C a(\sim 1.2)$ for viscous oil; however, for ethanol, the correlation was valid only for $\mathrm{Ca}<0.03$. The authors attributed the deviation beyond this Capillary number to inertial effects, as further confirmed in numerical studies by Giavedoni and Saita [12] and Heil [13].

Many numerical studies to date have contributed to improved understanding of two-phase slug flow in small channels [12-24]. Kreutzer et al. [21] investigated the influence of both the Capillary and Reynolds numbers on film thickness, pressure drop, and liquid flow patterns. The 
variation of film thickness at the middle of the slug, and a correlation to predict the pressure drop across the slug, were obtained for $0.002<C a<0.04$ and $0<\operatorname{Re}<900$. Gupta et al. [22] compared the volume of fluid (VOF) and level set (LS) interface tracking methods for simulating liquid-gas slug flow and heat transfer; both methods provided physically accurate results. The VOF method required a higher grid resolution, and the LS method a smaller time step, to accurately predict the flow field. Recently, Magnini et al. [23,24] investigated microchannel flow boiling in this regime, and found evaporation from the liquid film to be the dominant mechanism of heat transfer. More detailed accounts of the development of theoretical and numerical models can be found in recent review articles on the subject $[8,25,26]$. Most prior numerical studies, as summarized by Talimi et al. [26], were limited to $C a>\sim 0.005$, although flow behavior at smaller Capillary numbers is important to many applications of interest.

The continuum surface force (CSF) model [27] is one of the most popular methods for numerical treatment of surface tension forces, and is implemented in commercial computational fluid dynamics software packages [8,25,26]; however, it also induces unphysical spurious currents that hinder numerical investigation under small Capillary number conditions [28]. A large body of research has focused on developing methods to reduce these spurious currents (see review by Wörner [8]). Spurious currents are particularly limiting in the simulation of slug flow at small Capillary numbers. Gupta et al. [16] employed a structured square mesh in the interface region to minimize calculation inaccuracies that could induce spurious currents, but this approach can incur high computational expense. Mehdizadeh et al. [19] used dynamic mesh adaption to decrease the mesh size; the mesh was reconstructed every 5 time steps by refining the local mesh in the interface region and coarsening it in the bulk region. Horgue et al. [29] suggested that the spurious current could be reduced by using the High-Resolution Interface Capturing (HRIC) scheme employed in FLUENT [30], although the interface is more diffusive and the liquid film becomes thicker with this approach compared with the Geo-Reconstruct scheme [31]. Magnini et al. [23,24] coupled the VOF-CSF method with the Height Function method [32,33] for predicting the local surface shape to reduce spurious currents. A critical Capillary number exists below which these unphysical flows affect the simulation results. The critical Capillary number is usually on the order of $C a=0.01[28,34]$ to $C a=0.005$ [35].

A novel numerical treatment is proposed in the present work to reduce spurious currents in VOF-CSF simulations by imposing a moving reference frame to increase the local relative 
velocity magnitude near the interface. The dynamic interface of a gas slug is simulated as it is drawn into a liquid-filled channel. The numerical treatment is shown to suppress spurious currents at low Capillary numbers (as low as $C a \sim 0.003$ ) while preserving other physical features such as film thickness, gas slug velocity, and local liquid-phase flow patterns.

\section{Numerical method}

The simulations consider a circular channel initially filled entirely with liquid. At the start of the simulation, liquid is pumped out of one end of the channel with a constant velocity, $U_{l}$, and gas is drawn into the channel from ambient air on the other end to form an elongated gas slug (as shown in Fig. 1a). As the slug moves into the channel, a liquid film remains attached to the wall as the gas phase steadily displaces liquid. The volume of fluid-continuum surface force (VOFCSF) method is employed to simulate the fluid flow and track the liquid-gas interface in this slug flow scenario. A moving reference frame method is proposed to suppress the spurious currents inherent to this method. The numerical methods and governing equations are introduced in this section.

\subsection{Volume of fluid-continuum surface force method}

The VOF method [36] is an Eulerian approach for which the mesh is fixed and does not move with the interface. The two different phases are separated based on a so-called color function $C$, which presents the local volume fraction of the tracked phase. The volume fraction is then $C=1$ in cells filled with the tracked phase and $C=0$ in cells filled with the other phase. Cells with $0<$ $C<1$ are recognized as containing the interface. A transport equation for the volume fraction is solved to capture the motion of the interface.

$$
\frac{\partial C}{\partial t}+\vec{U} \cdot \nabla C=0
$$

With this treatment, the mass of the two phases is conserved and the two-phase flow problem can be solved by a single-phase formulation. For Newtonian fluids and laminar flow, the continuity and momentum equations are written as

$$
\begin{gathered}
\frac{\partial \rho}{\partial t}+\nabla(\rho \vec{U})=0 \\
\frac{\partial(\rho \vec{U})}{\partial t}+\nabla(\rho \vec{U} \cdot \vec{U})=-\nabla p+\nabla\left(\mu\left(\nabla \vec{U}+\nabla \vec{U}^{T}\right)\right)+F_{s}
\end{gathered}
$$


The fluid properties are determined from the volume-fraction-weighted average of the properties of the two phases. The surface tension force $F_{s}$, based on the CSF model [27], is treated as a volumetric body force, and is implemented in the cells around the interface where the gradient of volume fraction $\nabla C$ is not equal to zero.

$$
F_{s}=\sigma \kappa \delta_{s} \vec{n}=-\sigma \nabla \cdot\left(\frac{\nabla C}{|\nabla C|}\right)|\nabla C|
$$

With the above treatment, the surface tension force should be calculated to be normal to the interface using an accurate value of $\nabla C$; however, since the volume fraction $C$ in the interface region is not a continuous field, but is discretized with the mesh, it is difficult to obtain a completely accurate $\nabla C$ in VOF simulations. Small errors in the calculation of the volume fraction gradient may give rise to a surface tension force that is not strictly perpendicular to the interface, resulting in a spurious, tangential force component at the interface, or an error in the magnitude of surface tension. The calculated surface tension may then not be balanced by the pressure gradient, and can produce unphysical spurious currents in systems where the surface tension force is dominating, such as for example, when the Capillary number is small [28,35,37].

\subsection{Numerical solution procedure}

The numerical solution is obtained using the pressure-based finite volume scheme implemented in the software package ANSYS FLUENT [40]. Pressure-velocity coupling is accomplished through the PISO algorithm. The Green-Gauss node-based method is employed for accurately calculating scalar gradients and the Geo-Reconstruct method for volume-fraction discretization keeps the interface sharply resolved. The PRESTO and QUICK schemes are employed for discretization of pressure and momentum, respectively. A constant time step value is set in the range of $5 \times 10^{-8} \mathrm{~s}$ to $3 \times 10^{-7} \mathrm{~s}$ for each different case to ensure that the global Courant number is always smaller than 0.08 .

The 2D axisymmetric simulation domain has a channel length $L$ that is 40 times the channel diameter $D$. The mesh is shown in Fig. 1b. The cell length along the flow direction is uniformly $0.025 \mathrm{D}$. In the radial direction, the mesh is divided into three regions. In regions around the axis of the channel ( $0 D$ to $0.38 D$ from the axis), the radial length of the cell is uniform $(0.02375 D)$ and forms nearly square mesh cells. In an intermediate region between the axis and the channel wall $(0.38 D$ to $0.4865 D$ from the axis, 13 cells), the mesh is gradually refined in the radial direction approaching the wall. At the region adjacent to the wall $(0.4865 D$ to $0.5 D$ from 
the axis), the mesh is uniformly divided into 4 cells. For the lowest Capillary number investigated $(\mathrm{Ca}=0.0029)$, each cell in this wall-adjacent region is bisected in both directions (into four cells each) to ensure that the liquid film region always contains more than 5 cells in the radial direction, as recommended by Gupta et al. [16]. A total of 52,800 or 57,600 rectangular cells were included in the simulation domain depending on the liquid film thickness, with a maximum aspect ratio of 7.4. While this aspect ratio is not ideal for minimizing spurious currents as compared to a square mesh [16], the proposed numerical treatment for spurious current suppression allows for this larger aspect ratio to be used. Maintaining the cells square throughout the domain would increase the overall mesh size by a factor of $\sim 44$ relative to the present nonuniform mesh to resolve the liquid film region $(\sim 0.014 D$ at $C a=0.0029)$ with 5 cells through the film thickness.

The reference frame is set to move opposite to the gas slug at a velocity $U_{\text {ref }}$ to artificially induce a high velocity (relative to the reference frame) in the channel, and thus suppress the spurious current. Relative to the moving reference, the velocity in the channel $U_{r}$, becomes $U_{o}+$ $U_{r e f}$ and the pumping velocity $U_{l, r}$ becomes $U_{l, o}+U_{r e f}$. Correspondingly, the static wall must be assigned a velocity $U_{\text {ref }}$ with the direction of the gas slug in the moving reference frame.

The rationale for the present approach to spurious-current suppression may be understood by analyzing Eq. (3), as also explained in Section 3.2. The spurious currents arise as a result of an error in the surface tension force $F_{s}$ in Eq. (3) which cannot be balanced by the pressure gradient $\nabla p$; it must instead be balanced by the velocity terms. The surface tension force $F_{s}$ calculated from Eq. (4), and any associated errors, are independent of the velocity $U$ and of the moving reference frame. The moving reference frame increases the magnitude of the velocity terms in Eq. (3). As result, the influence of the errors in the surface tension term $F_{s}$ become relatively weaker compared with the velocity terms, leading to the spurious current suppression.

\section{Results and discussion}

The potential detrimental influence of spurious currents is first demonstrated by simulating slug flow in a static reference frame at a low Capillary number $(C a=0.0029)$. This problem is then simulated with the proposed moving reference frame to demonstrate suppression of these currents. To further demonstrate that other characteristic flow regime traits are not adversely affected by the present numerical treatment, flow circulation in the liquid region ahead of the gas 
slug is compared against results in the literature. The technique is then employed for another case $(C a=0.044)$ to illustrate the importance of increasing the near-interface relative velocity magnitude even for higher Capillary numbers.

\subsection{Moving reference frame method for spurious current suppression}

An air-water system is considered with fluid properties as shown in Table 1. The channel diameter $D$ is set at $0.715 \mathrm{~mm}$ and the liquid pumping velocity $U_{l, 0}$ is chosen to be $0.21 \mathrm{~m} / \mathrm{s}$ to allow a comparison between the present simulation and recently published experiments [38] at $C a=\mu U_{l, 0} / \sigma=0.0029$.

When simulated using a static reference frame, spurious currents appear near the interface as the gas slug progresses along the channel, as shown in Fig. 2. At time of $t=2.1 \mathrm{~ms}$ after the gas is initially drawn into the channel (Fig. 2a), these spurious currents reach the same order of magnitude as the liquid pumping velocity in the channel (Fig. 2c). These unphysical flows intensify over the next $0.3 \mathrm{~ms}$ until the gas-liquid interface is ultimately broken (Fig. 2b and 2c). A series of spurious droplets are entrained into the gas slug at the central axis, rendering a false solution; the simulation is ceased at this time.

The same problem is then modeled with the proposed moving reference method. All conditions and simulation parameters are identical, except that the reference frame is set with a velocity $U_{r e f}=0.6 \mathrm{~m} / \mathrm{s}$ opposite the flow direction. The simulation results are summarized in Fig. 3. At a time when the flow is fully developed in the channel (i.e., a constant interface profile is achieved), long after spurious currents had disrupted the interface for the static reference frame simulation, the liquid-gas interface profile and nearby velocities are respectively shown in Fig. $3 \mathrm{a}$ and Fig. 3b; the spurious currents are clearly absent. The calculated fully developed film thickness, $\delta=10.7 \mu \mathrm{m}$, closely agrees with the measured value in [38] of $\delta=10.2 \mu \mathrm{m}$. The calculated absolute velocity of the gas slug is $0.223 \mathrm{~m} / \mathrm{s}$. This agrees with the value evaluated $\left(U_{S, 0}=0.222 \mathrm{~m} / \mathrm{s}\right)$ using the correlation by Fairbrother and Stubbs [39]:

$$
\frac{U_{S, 0}-U_{l, 0}}{U_{l, 0}}=\left(\frac{\mu U_{S, 0}}{\sigma}\right)^{\frac{1}{2}}
$$

Detailed interrogation of the flow field in the liquid region ahead of the leading interface of the gas slug (observed with a reference frame travelling with the slug) reveals flow circulation zones (Fig. 3c) which are pertinent to micro-reactor systems [5-8] and heat transfer [18,19,23-26]. The liquid moves faster than the gas slug in the center region of the channel, and therefore has a 
relative velocity with respect to the slug in a direction away from the interface. Conversely, in the wall region, the liquid is drawn toward the circumferential liquid film. This circulation flow pattern is well-captured in the present simulation, with no sign of spurious velocities around the liquid-gas interface.

In order to further demonstrate that the present numerical treatment does not sacrifice physical resolution while suppressing spurious currents, the circulating flow field ahead of the leading gas slug interface is investigated for slug flow simulations with both static and moving reference frames at $C a=0.05$ and $R e=1$. The velocity of the moving reference frame is chosen to be the same as the pumping velocity, i.e., $U_{r e f}=U_{l, 0}$. To quantitatively compare with the numerical investigation by Heil [13] at $C a=0.05$ and $R e \rightarrow 0$, the simulations are performed in two dimensions (not axisymmetric). The calculated flow fields are shown in Fig. 4. Considering that this case represents a Capillary number greater than the typical critical Capillary number value, it is expected that a static reference frame would not induce spurious currents, as confirmed in Fig. 4a. The proposed moving reference frame method yields the same result as the static reference method, confirming this liquid flow field is accurately represented. The predicted circulation flow pattern, normalized vorticity field, and maximum normalized absolute vorticity (4.5) as shown in Fig. 4b match the results in the literature [13].

\subsection{Spurious current suppression mechanism}

The mechanism by which spurious currents are suppressed by setting the reference frame to move opposite the two-phase flow direction is discussed here. The relative velocity near the interface is increased in this moving reference frame, thus masking the small numerical errors in the calculated surface tension term that give rise to spurious velocities. This effect can be illustrated by considering a case with a moderate Capillary number.

An air-ethanol system with a channel diameter of $0.995 \mathrm{~mm}$ and liquid pumping velocity of $0.843 \mathrm{~m} / \mathrm{s}$ is considered. These parameters match the experimental conditions considered by Han and Shikazono [38], leading to a $C a=0.044$. The simulation result when using a static reference frame is shown in Fig. 5a. Even though the Capillary number is moderately large (i.e., the liquid superficial velocity is high), since the liquid film is very thin in some regions of the flow, the velocity magnitude near the liquid-gas interface remains small. In the inset of Fig. 5a, it is seen that spurious currents can still be formed in regions of the liquid film that become sufficiently thin as gas is drawn into the channel. In this case, to induce a higher relative velocity 
at the liquid film region, the reference frame can even be set to move in the same direction as the liquid flow (rather than moving in a direction opposite that of the flow). The spurious current is suppressed for $U_{r e f}=-0.8 \mathrm{~m} / \mathrm{s}$ (Fig. 5b) and the calculated thickness of the liquid film is $67 \mu \mathrm{m}$, which agrees with the experimentally measured value $63.2 \mu \mathrm{m}$ in [38].

This two-phase slug flow case clearly demonstrates that the critical mechanism by which the proposed moving reference frame treatment suppresses spurious currents is an increase of the relative velocity magnitude near the interface. For high Capillary number flows, setting the reference frame to move in the flow direction at the same velocity as the vapor slug will also avoid the presence of spurious currents in the liquid thin film region by increasing this nearinterface relative velocity; such a reference frame has been widely used by researchers $[1,8,18,23-26]$, but the purpose has been to reduce the size of the simulation domain rather than the serendipitous benefit of spurious-current suppression demonstrated here. By tuning the relative direction of the moving reference frame with respect to the flow direction as a function of the Capillary number, it is thereby possible to simultaneously suppress spurious currents and minimize the computational expense (where low $\mathrm{Ca}$ demands the largest computational domain with $U_{r e f}>0$, and high $C a$ requires the smallest domain at $U_{r e f}=-U_{S, 0}$ )

\section{Conclusions}

A novel and straightforward numerical treatment is proposed to suppress spurious currents in VOF-CSF simulation of two-phase slug flow at small Capillary numbers. An artificial relative reference frame is specified with motion opposite to the flow direction to suppress spurious currents formed by the inaccurately calculated surface tension force. The increase of the local relative velocity magnitude near the interface is demonstrated to be the key mechanism for spurious-current suppression.

A comparison of simulations performed with and without this treatment shows that spurious currents are eliminated at $C a=0.0029$; liquid film thickness, gas slug velocity, and liquid-phase circulation near the leading gas slug interface are preserved and agree with the literature. This demonstrates that the present moving reference frame method does not influence real physical phenomena of interest while suppressing unphysical spurious velocities.

\section{Acknowledgment}


This material is based upon work supported by the Defense Advanced Research Projects Agency (DARPA) Microsystems Technology Office's (MTO) Intrachip/Interchip Enhanced Cooling (ICECool) Fundamentals program under Cooperative Agreement No. HR0011-13-2-0010.

The content of the information does not necessarily reflect the position or the policy of the Government, and no official endorsement should be inferred. Distribution Statement A - Approved for public release; distribution unlimited.

\section{References}

1. V. Talimi, Y.S. Muzychka, and S. Kocabiyik, Numerical Simulation of the Pressure Drop and Heat Transfer of Two Phase Slug Flows in Microtubes using Moving Frame of Reference Technique, Int. J. Heat Mass Transfer, vol. 55, pp. 6463-6472, 2012.

2. C. Narayanan, and D. Lakehal, Two-Phase Convective Heat Transfer in Miniature Pipes under Normal and Microgravity Conditions, J. Heat Transfer, vol. 130, pp. 074502, 2008.

3. J.R. Thome, V. Dupont, and A. Jacobi, Heat Transfer Model for Evaporation in Microchannels Part I: Presentation of the Model, Int. J. Heat Mass Transfer, vol. 47, pp. 3387-3401, 2004.

4. T. Harirchian, S.V. Garimella, Flow Regime-Based Modeling of Heat Transfer and Pressure Drop in Microchannel Flow Boiling, Int. J. Heat Mass Transfer, vol. 55, pp. 1246-1260, 2012.

5. T. Can, M. Liu, and Y. Xu, 3-D Numerical Simulations on Flow and Mixing Behaviors in Gas-Liquid-Solid Microchannels, AICHE J., vol. 59, pp. 1934-1951, 2013.

6. M.T. Kreutzer, F. Kapteijn, J.A. Moulijn, and J.J. Heiszwolf, Multiphase Monolith Reactors: Chemical Reaction Engineering of Segmented Flow in Microchannel, Chem. Eng. Sci., vol. 60, pp. 5895-5916, 2005. 
7. R. M. Santos, M. Kawaji, Numerical Modeling and Experimental Investigation of GasLiquid Slug Formation in a Microchannel T-Junction, Int. J. Multiphase Flow, vol. 36, pp. 314-323, 2010.

8. M. Wörner, Numerical Modeling of Multiphase Flows in Microfluidics and Micro Process Engineering: A Review of Methods and Applications, Microfluid. Nanofluid., vol. 12, pp. 841-886, 2012.

9. F.P. Bretherton, The Motion of Long Bubbles in Tubes, J. Fluid Mech., vol. 10, pp. 166-188, 1961.

10. P. Aussillous, and D. Quéré, Quick Deposition of Fluid on the Wall of a Tube, Phys. Fluid., vol. 12, pp. 2367-2371, 2000.

11. G.I. Taylor, Deposition of Viscous Fluid on the Wall of a Tube, J. Fluid Mech., vol. 10, pp. 161-165, 1961.

12. M.D. Giavedoni, and F.A. Saita, The Axisymmetric and Plane Cases of a Gas Phase Steadily Displacing a Newtonian Liquid: A Simultaneous Solution of the Governing Equations, Phys. Fluid., vol. 9, pp. 2420-2428, 1997.

13. M. Heil, Finite Reynolds Number Effects in the Bretherton Problem, Phys. Fluid., vol. 13, pp. 2517-2521, 2001.

14. A.L. Hazel, M. Heil, The Steadty Propagation of a Semi-Infinite Bubble into a Tube of Elliptical or Rectangular Cross-Section, J. Fluid Mech., vol. 470, pp. 91-114, 2002.

15. T. Taha, Z.F. Cui, CFD Modelling of Slug Flow inside Square Capillaries, Chem. Eng. Sci., vol. 61, pp. 665-675, 2006.

16. R. Gupta, D.F. Fletcher, B.S. Haynes, On the CFD Modelling of Taylor Flow in Microchannels, Chem. Eng. Sci., vol. 64, pp. 2941-2950, 2009. 
17. Y. Chen, R. Kulenovic, R. Mertz, Numerical Study on the Formation of Taylor Bubbles in Capillary Tubes, Int. J. Therm. Sci., vol. 48, pp. 234-242, 2009.

18. A.N. Asadolahi, R. Gupta, D.F. Fletcher, and B.S. Haynes, CFD Approaches for the Simulation of Hydrodynamics and Heat Transfer in Taylor Flow, Chem. Eng. Sci., vol. 66, pp. 5575-5584, 2011.

19. A. Mehdizadeh, S.A. Sherif, and W.E. Lear, Numerical Simulation of Thermofluid Characteristics of Two-Phase Slug Flow in Microchannels, Int. J. Heat Mass Transfer, vol. 54, pp. 3457-3465, 2011.

20. U. Olgac, M. Muradoglu, Effects of Surfactant on Liquid Film Thickness in the Bretherton Problem, Int. J. Multiphase Flow, vol. 48, pp. 58-70, 2013.

21. M.T. Kreutzer, F. Kapteijn, J.M. Moulijn, C.R. Kleijn, and J.J. Heiszwolf, Inertial and Interfacial Effects on Pressure Drop of Taylor Flow in Capillaries, AICHE J., vol. 51, pp. 2428-2440, 2005.

22. R. Gupta, D.F. Fletcher, B.S. Haynes, CFD Modelling of Flow and Heat Transfer in the Taylor Flow Regime, Chem. Eng. Sci., vol. 65, pp. 2094-2107, 2010.

23. M. Magnini, B. Pulvirenti, and J.R. Thome, Numerical Investigation of Hydrodynamics and Heat Transfer of Elongated Bubbles during Flow Boiling in Microchannel, Int. J. Heat Mass Transfer, vol. 59, pp. 451-471, 2013.

24. M. Magnini, B. Pulvirenti, and J.R. Thome, Numerical Investigation of the Influence of Leading and Sequential Bubbles on Slug Flow Boiling within a Microchannel, Int. J. Therm. Sci., vol. 71, pp. 36-52, 2013. 
25. R. Gupta, D.F. Fletcher, B.S. Haynes, Taylor Flow in Microchannels: A Review of Experimental and Computational Work, J. Computational Multiphase Flows, vol. 2, pp. 1-32, 2010.

26. V. Talimi, Y.S. Muzychka, and S. Kocabiyik, A Review on Numerical Studies of Slug Flow Hydrodynamics and Heat Transfer in Microtubes and Microchannels, Int. J. Multiphase Flow, vol. 39, pp. 88-104, 2012.

27. J.U. Brackbill, D.B. Kothe, C. Zemach, A Continuum Method for Modeling Surface Tension, J. Comput. Phys., vol. 100, pp. 335-354, 1992.

28. B. Lafaurie, C. Nardone, R. Scardovelli, S. Zaleski, G. Zanetti, Modelling Merging and Fragmentation in Multiphase Flows with SURFER, J. Comput. Phys., vol. 113, pp. 134-147, 1994.

29. P. Horgue, F. Augier, M. Quintard, M. Prat, A Suitable Parametrization to Simulate Slug Flows with the Volume-Of-Fluid Method, Comptes Rendus Mecanique, vol. 340, pp. 411419, 2012.

30. S. Muzaferija, M. Peric, P. Sames, T. Schellin, A Two-Fluid Navier-Stokes Solver to Simulate Water Entry, Proc. 22nd Symp. Naval Hydrodyn., Washington, 1998.

31. D. Youngs, Time-Dependent Multi-Material Flow with Large Fluid Distortion, in K.W. Morton, M.J. Baines (Eds.), Numerical Methods for Fluid Dynamics, pp.273-285, Academic Press, London, 1982.

32. M. Malik, E.S.-C. Fan, M. Bussmann, Adaptive VOF with Curvature-Based Refinement, Int. J. Numer. Method. Fluid., Vol. 55, pp. 693-712, 2007. 
33. J. Hernandez, J. Lopez, P. Gomez, C. Zanzi, F. Faura, A New Volume of Fluid Method in Three Dimensions-Part I: Multidimensional Advection Method with Face-Matched Flux Polyhedra, Int. J. Numer. Method. Fluid., Vol. 58, pp. 897-921, 2008.

34. T. Abadie, J. Aubin, D. Legendre, C. Xuereb, Hydrodynamics of Gas-Liquid Taylor Flow in Rectangular Microchannels, Microfluid. Nanofluid., vol. 12, pp. 355-369, 2012.

35. J.B. Dupont, D. Legendre, Numerical Simulation of Static and Sliding Drop with Contact Angle Hysteresis, J. Comput. Phys., vol. 229, pp. 2453-2478, 2010.

36. C.W. Hirt, B.D. Nichols, Volume of Fluid (VOF) Method for the Dynamics of Free Boundaries, J. Comput. Phys., vol. 39, pp. 201-225, 1981.

37. D.J.E. Harvie, M.R. Davidson, M. Rudman, An Analysis of Parasitic Current Generation in Volume of Fluid Simulations, Appl. Math. Modelling, vol. 30, pp. 1056-1066, 2006.

38. Y. Han, and N. Shikazono, Measurement of the Liquid Film Thickness in Micro Tube Slug Flow, Int. J. Heat Fluid Flow, vol. 30, pp. 842-853, 2009.

39. F. Fairbrother, A.E. Stubbs, Studies in Electroendosmosis - VI. The Bubble Tube Method of Measurement, J. Chem. Soc., vol. 1, pp. 527-529, 1935.

40. Fluent, ANSYS FLUENT 14.0: User's Guide, ANSYS-Fluent Inc., Lebanon, N.H., 2009. 


\section{List of Tables}

Table 1. Fluid properties. 
Table 1. Fluid properties.

\begin{tabular}{lccc}
\hline Property & Water & Ethanol & Air \\
\hline Density, $\rho\left(\mathrm{kg} / \mathrm{m}^{3}\right)$ & 998 & 789 & 1.225 \\
Viscosity, $\mu(\mathrm{kg} / \mathrm{m} \mathrm{s})$ & $1.001 \times 10^{-3}$ & $1.196 \times 10^{-3}$ & $1.79 \times 10^{-5}$ \\
Surface tension, $\sigma(\mathrm{N} / \mathrm{m})$ & 0.0727 & 0.0228 & \\
\hline
\end{tabular}




\section{List of Figures}

Fig. 1 (a) Schematic illustrations of a gas slug traveling through a channel and (b) the associated simulation domain, boundary conditions, and mesh with a reference frame set moving opposite to the flow direction.

Fig. 2 Simulation of slug flow with a static reference frame (air-water, $D=0.715 \mathrm{~mm}, U l_{, 0}=$ $0.21 \mathrm{~m} / \mathrm{s}, C a=0.0029$ ). (a) The instantaneous phase profiles are shown at $t=2.1 \mathrm{~ms}$; (b) spurious currents break the interface and produce droplets entrained in the gas phase from $t=$ $2.4 \mathrm{~ms}$. (c) The velocity fields in the channel are shown at $t=2.1 \mathrm{~ms}$ (left) and $t=2.4 \mathrm{~ms}$ (right).

Fig. 3 Simulation of slug flow with a moving reference frame (air-water, $D=0.715 \mathrm{~mm}, U l_{, 0}$ $=0.21 \mathrm{~m} / \mathrm{s}, C a=0.0029$, Uref $=0.6 \mathrm{~m} / \mathrm{s}$ ). The fully-developed (a) phase profiles and (b) absolute flow field in the channel are shown; (c) flow near the interface is observed with a reference frame moving with the gas slug.

Fig. 4 Comparison of the flow fields calculated with (a) a static reference frame $(\mathrm{Ca}=0.05$, $R e=1)$ and with (b) the moving reference frame method $\left(U_{r e f}=U_{l, 0}, C a=0.05, R e=1\right)$

Fig. 5 Simulation of slug flow for an air-ethanol system $\left(D=0.995 \mathrm{~mm}, U_{l, 0}=0.843 \mathrm{~m} / \mathrm{s}, C a\right.$ $=0.044)$ : (a) Instantaneous phase profiles and spurious currents (inset) near the static wall are shown for a static reference frame at $t=3.6 \mathrm{~ms}$; (b) with the moving reference frame method $\left(U_{r e f}=-0.8 \mathrm{~m} / \mathrm{s}\right)$; the spurious current is suppressed and does not affect the fully developed phase profiles. 

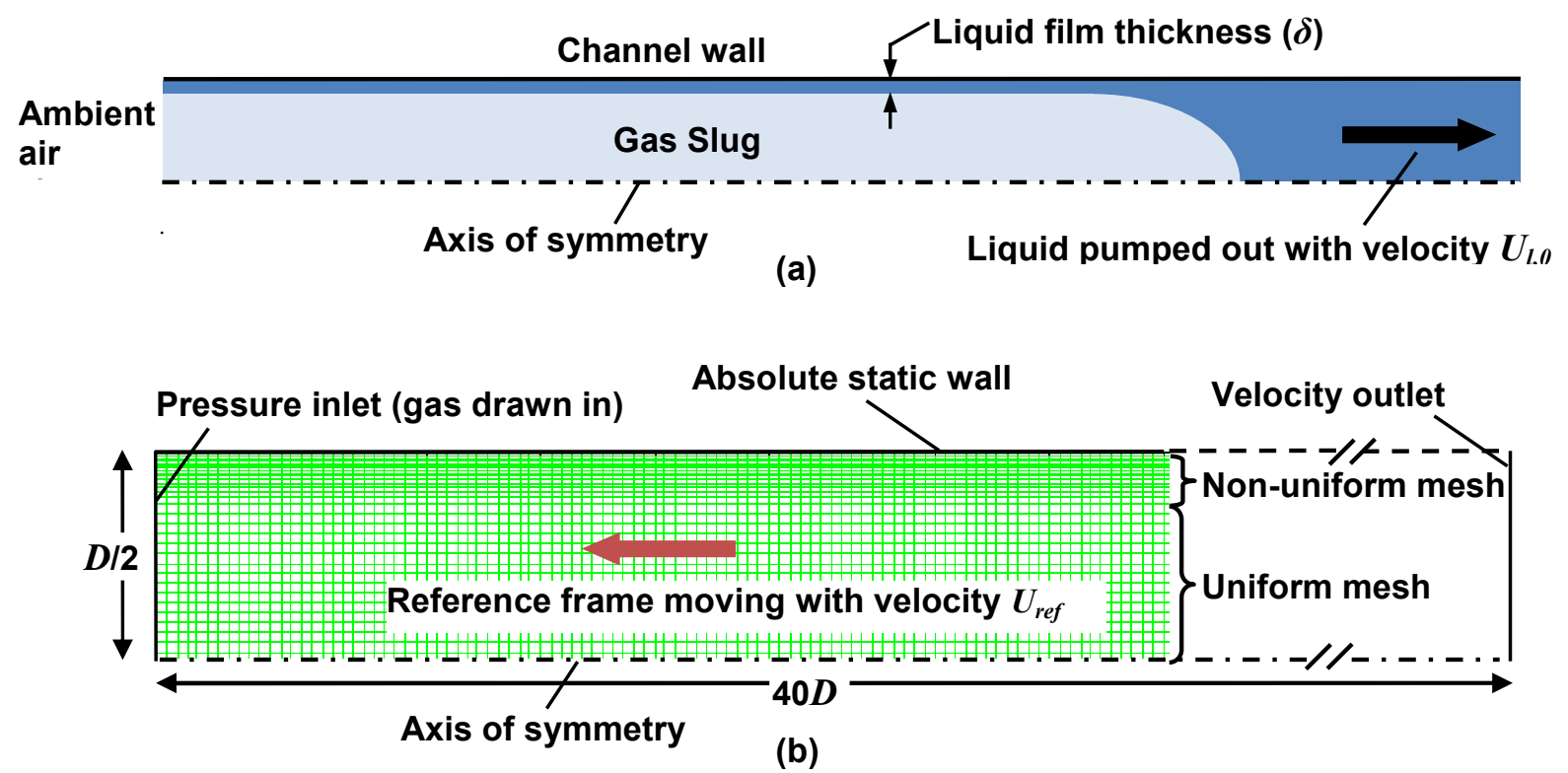

Fig. 1 (a) Schematic illustrations of a gas slug traveling through a channel and (b) the associated simulation domain, boundary conditions, and mesh with a reference frame set moving opposite to the flow direction. 


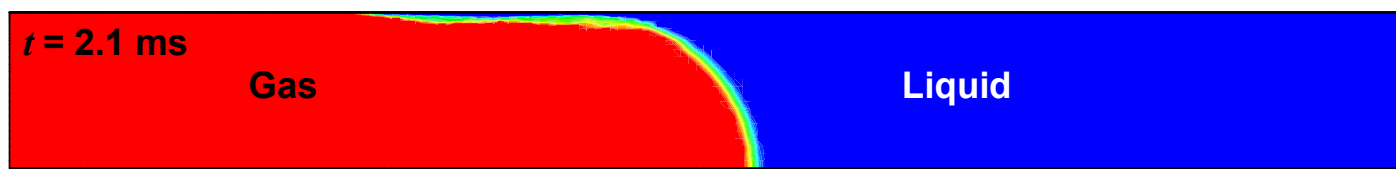

(a)
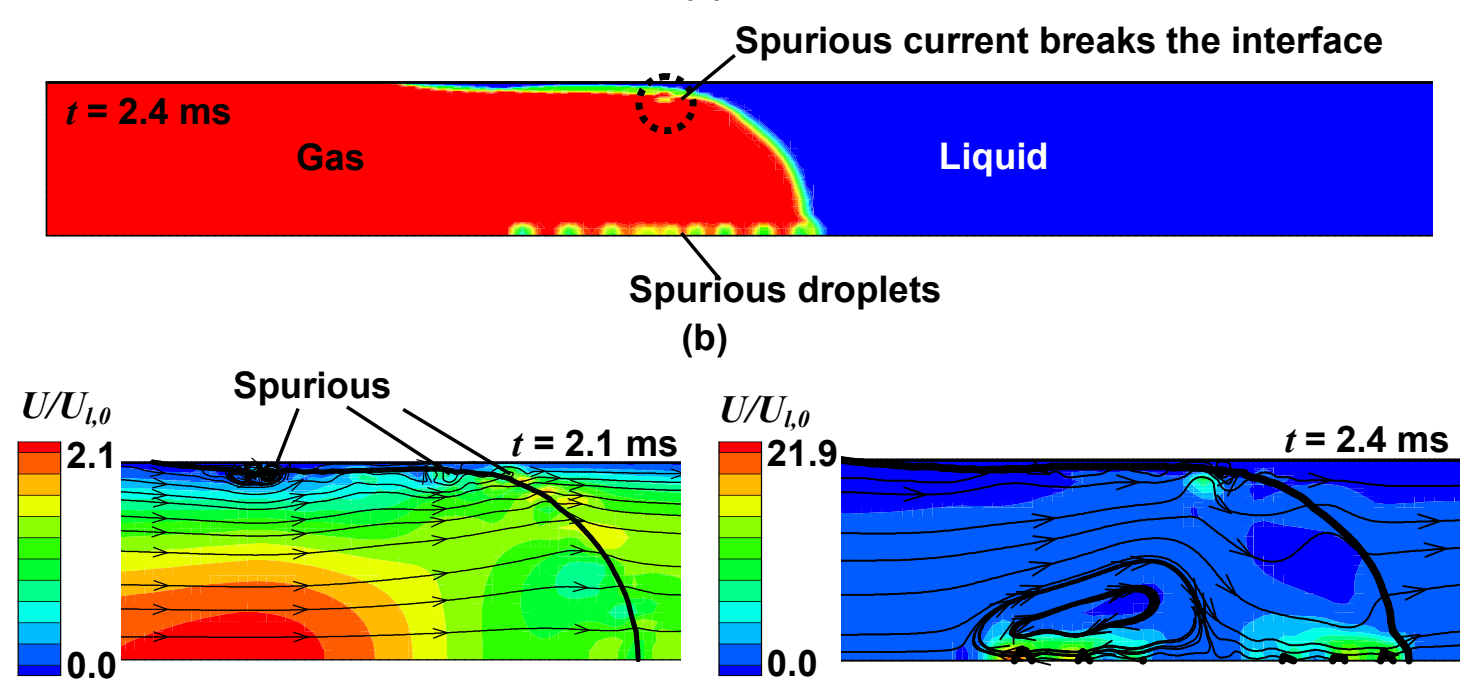

(c)

Fig. 2 Simulation of slug flow with a static reference frame (air-water, $D=0.715 \mathrm{~mm}, U_{l, 0}=0.21$ $\mathrm{m} / \mathrm{s}, C a=0.0029$ ). (a) The instantaneous phase profiles are shown at $t=2.1 \mathrm{~ms}$; (b) spurious currents break the interface and produce droplets entrained in the gas phase from $t=2.4 \mathrm{~ms}$. (c) The velocity fields in the channel are shown at $t=2.1 \mathrm{~ms}$ (left) and $t=2.4 \mathrm{~ms}$ (right). 


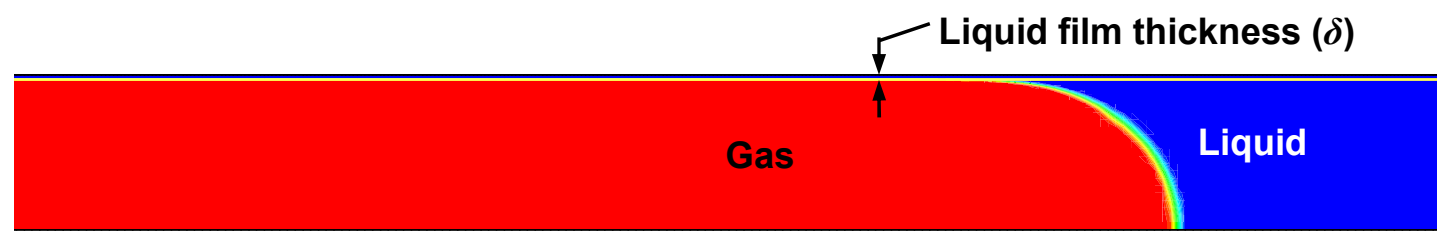

(a)

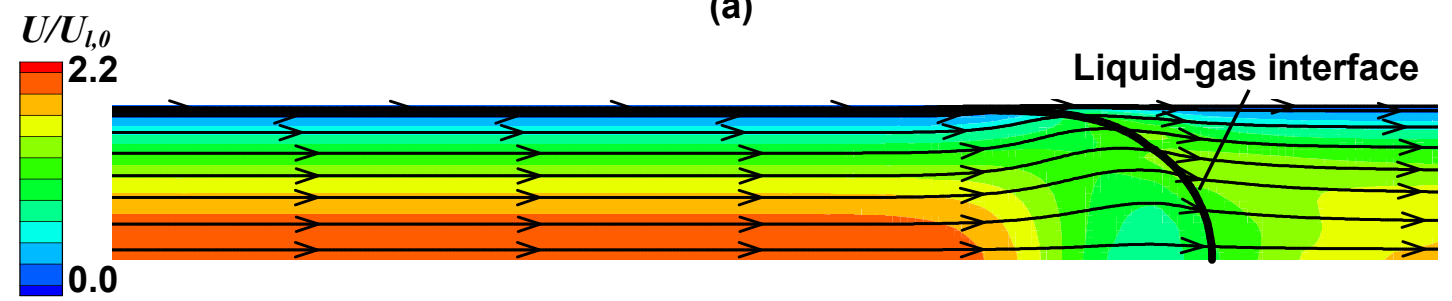

(b)

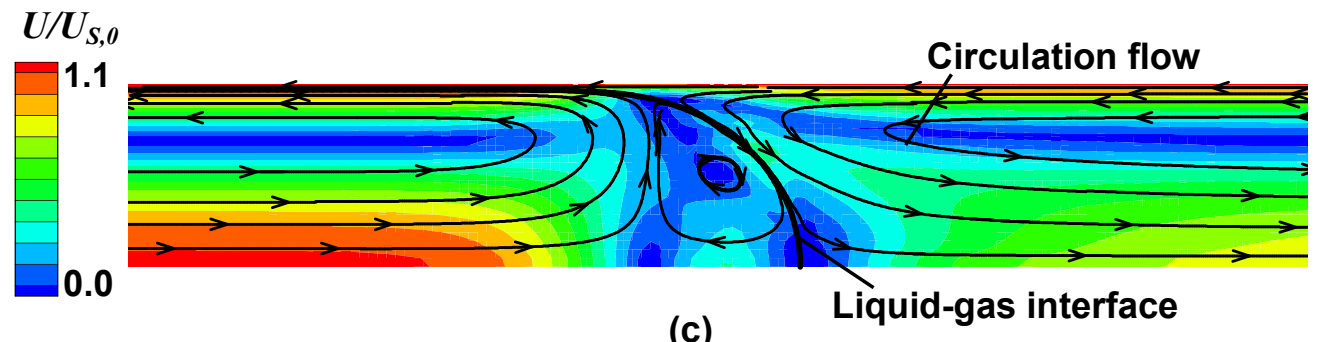

(c)

Fig. 3 Simulation of slug flow with a moving reference frame (air-water, $D=0.715 \mathrm{~mm}, U_{l, 0}=$ $0.21 \mathrm{~m} / \mathrm{s}, C a=0.0029, U_{r e f}=0.6 \mathrm{~m} / \mathrm{s}$ ). The fully-developed (a) phase profiles and (b) absolute flow field in the channel are shown; (c) flow near the interface is observed with a reference frame moving with the gas slug. 


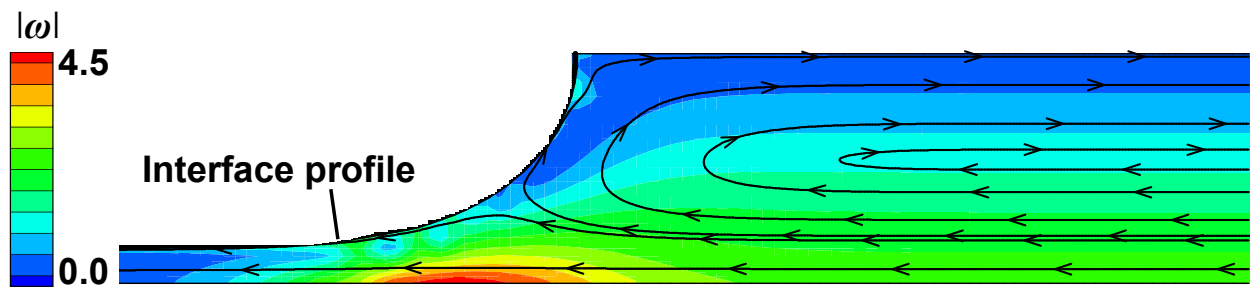

(a)

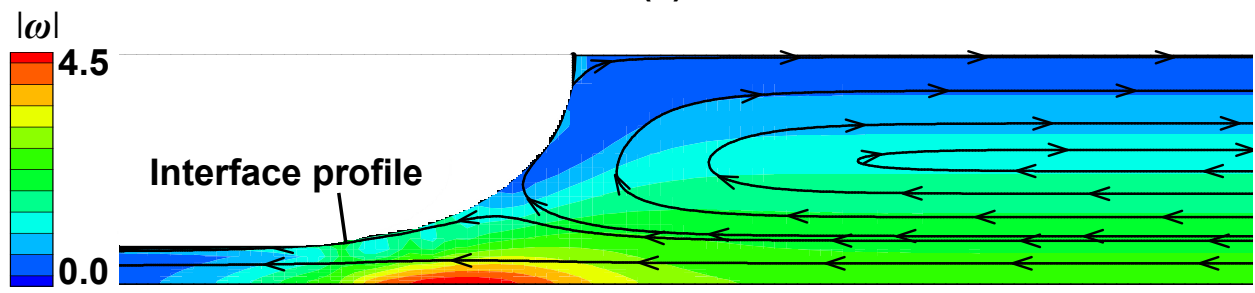

(b)

Fig. 4 Comparison of the flow fields calculated with (a) a static reference frame $(\mathrm{Ca}=0.05, \operatorname{Re}=$ 1) and with (b) the moving reference frame method ( $\left.U_{r e f}=U_{l, 0}, C a=0.05, R e=1\right)$. The nondimensional vorticity magnitude in (a) and (b) is normalized by gas slug velocity and channel half width as in [13]. 


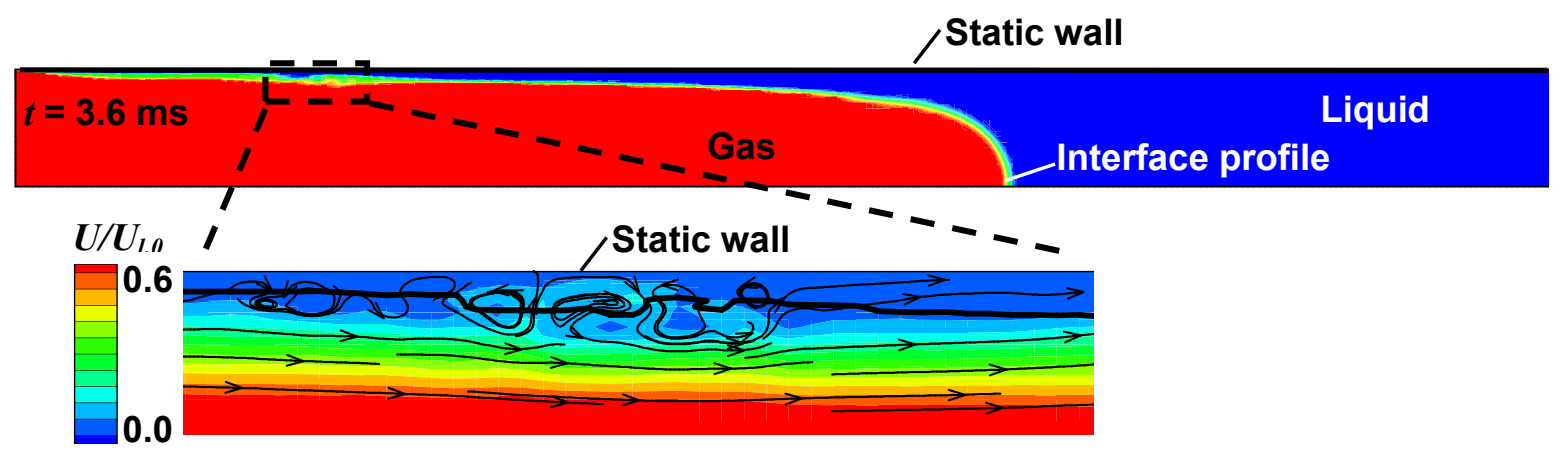

(a)

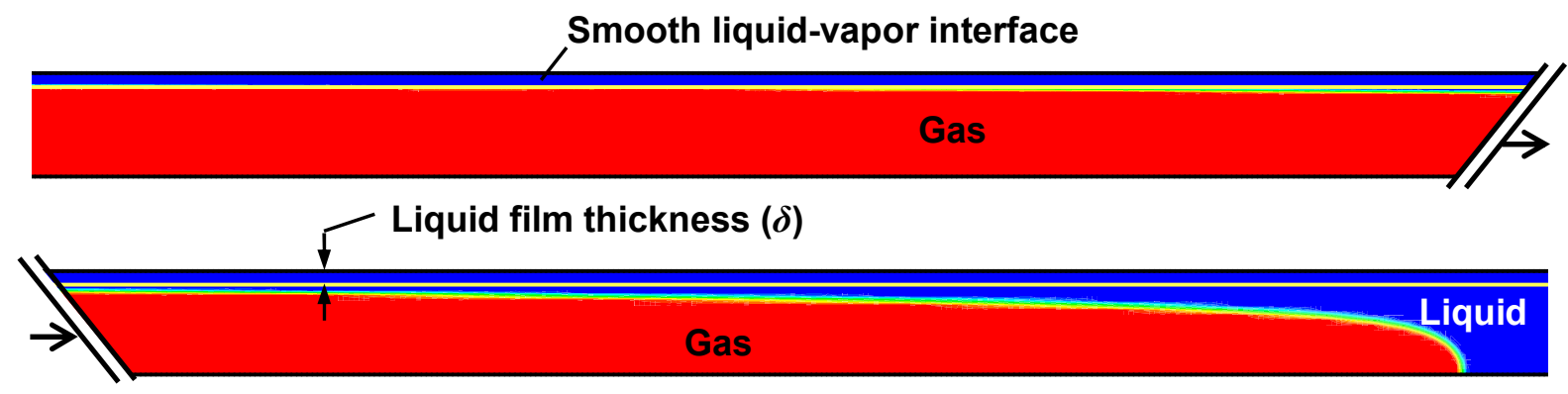

(b)

Fig. 5 Simulation of slug flow for an air-ethanol system $\left(D=0.995 \mathrm{~mm}, U_{l, 0}=0.843 \mathrm{~m} / \mathrm{s}, C a=\right.$ 0.044): (a) Instantaneous phase profiles and spurious currents (inset) near the static wall are shown for a static reference frame at $t=3.6 \mathrm{~ms}$; (b) with the moving reference frame method $\left(U_{\text {ref }}=-0.8 \mathrm{~m} / \mathrm{s}\right.$ ); the spurious current is suppressed and does not affect the fully developed phase profiles. 